\title{
NEAR INFRARED INTERFEROMETRY OF THE GALILEAN SATELLITES
}

\section{R. Howell}

Steward Observatory and Planetary Sciences Dept., University of Arizona

\section{ABSTRACT}

Some of the Galilean satellites show evidence of two distinct surface types, a high albedo frost and a dark, presumably silicate material We are beginning a program to map the spatial distribution of these components with a Michelson spatial interferometer. This will be done both in and next to the $2.0 \mu \mathrm{m}$ frost absorption band. While the mapping program is just beginning, this investigation has already yielded new information on the wavelength dependence of seeing in the infrared.

\section{INTRODUCTION}

The Galilean satellites became the first astronomical bodies to be investigated by spatial interferometry when in the 1890's Michelson measured their diameters ${ }^{1}$ The project we are undertaking is an attempt to apply infrared technology in an experiment very similar to Michelson's. In preparing for this we have obtained new information on the character of infrared seeing and that data will also be presented.

It has been known for six years that Europa, Ganymede, and perhaps Callisto are partially covered by water frost ${ }^{2-4}$ There is considerable evidence that there are two distinct types of surfaces on these satellites-the high albedo frost and some dark material with an albedo of $\leq 1$ The frost and dark material cannot be mixed on a scale small enough to allow thermal equilibrium between them because the frost is estimated to be at $100^{\circ} \mathrm{K}$ from infrared spectroscopy while the temperature of the dark material must be $>150^{\circ} \mathrm{K} .{ }^{5}$ Any "dirty snow" should sublimate leaving a blanket of dark material. 6

The two component model is not only indicated by spectroscopy, it can also explain the character of the $10 \mu$ to $20 \mu$ photometry and the eclipse cooling curves The brightness temperature decreases from $10 \mu \mathrm{m}$ to $20 \mu \mathrm{m}$ 
in the manner expected for a body having two surface temperatures The percentage of the surface at each temperature is consistent with that indicated by spectroscopy 7 The model proposed by Morrison and Cruikshank, and by Hansen to explain the eclipse cooling curves is quite different. 8,9 When Ganymede and Callisto enter Jupiter's shadow, the thermal flux from them decreases rapidly at first but soon begins to level off. They proposed this was due to vertical structure, a thin layer of low thermal inertia material with a high conductivity subsurface. Specifically, they suggested the top layer was frost and the bottom rock or rock-ice. From their observations they placed limits on the amount of this subsurface that could be exposed, $5 \%$ for Ganymede and 1\% for Callisto This thin, almost universal frost layer, is inconsistent with spectroscopy However, another interpretation is possible If the proposed dark component has low thermal inertia while the frost's is high, then the steep drop in flux at the beginning of the eclipse would be due to the cooling of the dark component (which supplies most of the thermal flux) while the residual flux would be from the frost. Detailed calculations for Ganymede show this model is consistent with the other observations

While there is considerable evidence for this two component model, the spatial distribution is unknown. As mentioned the size scale is larger than $\mathrm{cm}$ size. There may be some structure on a planetary scale Visual observers have claimed the existence of polar caps on Europa and Ganymede and there is also variability with longitude, however, this was not evident in the Pioneer 10 images

\section{TECHNIQUE}

The purpose of this investigation is to determine if this two component structure exists on a planetary scale and if so what is the spatial distribution To do this we plan to obtain visibility curves along north-south and east-west baselines and at two wavelengths 0 ne is at $2.22 \mu \mathrm{m}$ where the

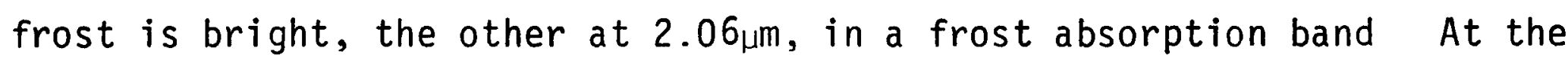
second wavelength the contrast between the frost and dark material is reduced, providing a check on the results obtained with the first filter 
From these observations it should be possible to determine conclusively the presence or absence of polar caps.

Figure 1 shows an extreme example of the type of effect expected. These are the visibility curves for an object with .9 albedo polar caps above $50^{\circ}$ and .1 albedo material below this since this contrast is larger than that expected, and since this model did not include the important effect of limb darkening, the expected result would be less dramatic. However, from the visibility curves, it should be possible to measure the contrast between the polar and equatorial regions, and the size of any caps which might exist. If the frost is not concentrated at the poles but still is concentrated in distinct regions, it should again be possible to estimate their size and albedo.

\section{INSTRUMENTATION}

The instrument being used has been described earlier at this meeting by $\mathrm{Dr}$. Low. For the past several years it has been used routinely at

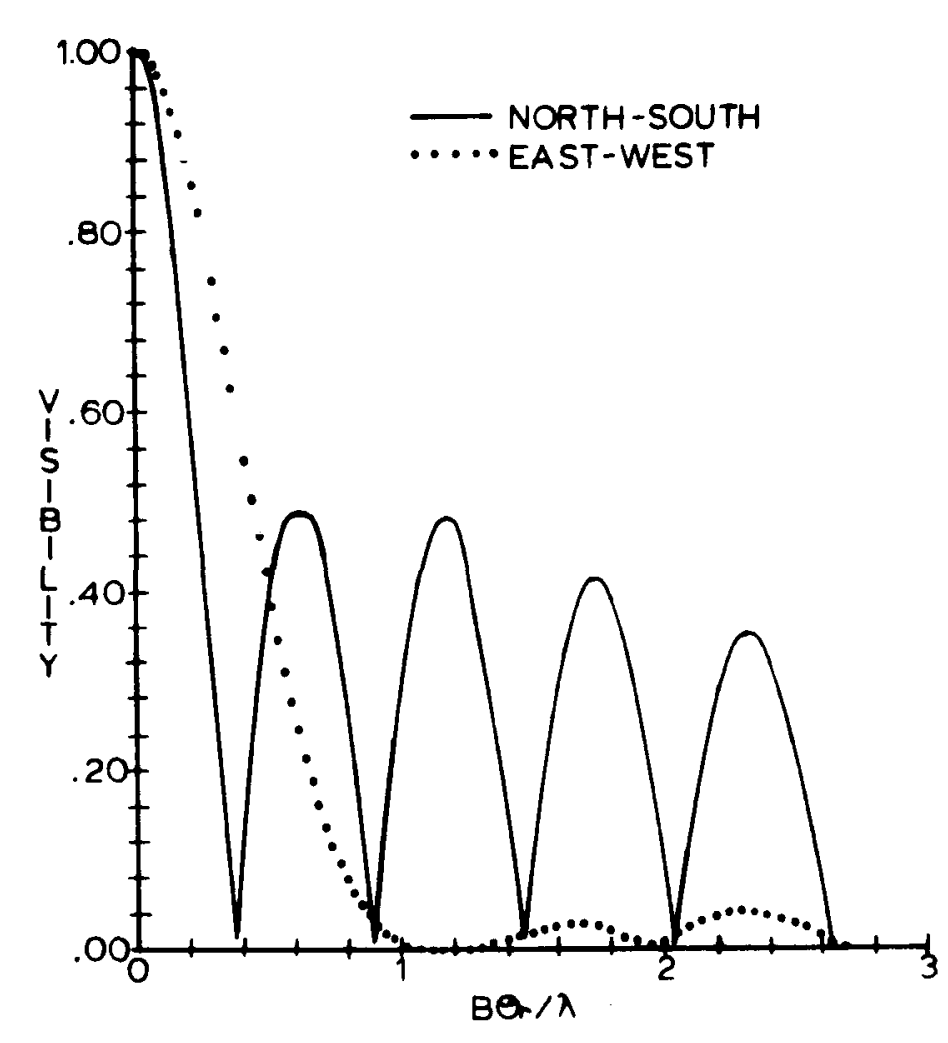

Figure 1. The visibility function of a planet with bright polar caps. B is the baseline and $\theta_{r}$ is the angular radius Limb darkening has been ignored so this is only an illustration of the type of effect expected 
wavelength from $5 \mu \mathrm{m}$ to $12 \mu \mathrm{m}$ to measure the visibilities of stars showing evidence of circumstellar shells, a topic to be presented by Dr McCarthy On a few occasions it has been used at $2 \mu \mathrm{m}$ and $3 \mu \mathrm{m}$. The extension of regular observations to shorter wavelengths necessitates both improvements in the instrument and a better understanding of the seeing which begins to degrade the performance at these wavelengths

\subsection{Modulation of the Interference Fringes}

The instrument produces a diffraction disk with the interference pattern superposed on it. The path length difference between beams can be changed and the standard observing procedure is to modulate it in a sawtooth pattern - a linear scan of one wavelength followed by a quick retrace. This scans the fringes across a mask in front of the detector and produces a sine wave whose phase is a measure of the position of the fringes A shift of $2 \pi$ in phase corresponds to a path length difference of one wavelength For bright objects the changes produced by seeing can be tracked over many wavelengths if they are sufficiently slow

\subsection{Signal Processing}

If the atmosphere did not introduce phase shifts it would be possible to phase detect this signal and one would obtain an estimate of the fringe amplitude whose precision was proportional to the signal to noise ratio at the detector. Unfortunately, this is not the case If in some characteristic time $T_{c}$ the atmosphere introduces path length difference of one wavelength then the signal will have a bandwidth of $\sim \frac{1}{T_{c}}$. To measure the amplitude one must measure the power (that is the mean ${ }^{c}$ square detector output) in this bandpass both with the signal present and with it absent The square root of the difference is proportional to the fringe amplitude. For weak signals, the signal to noise of the result is $\sim(s / n)^{2} \sqrt{T_{T}}$ where $s$ is the signal, $n$ is the noise per root hertz, and $T$ is the integration time

\subsection{Signal to Noise Ratio}

The liquid nitrogen cooled InSb detector that has been used in the past will be replaced this fall by a helium cooled InSb system. Such detectors 
have recently achieved NEP's of $7 \times 10^{-17}$ watts $/ \overline{\mathrm{hz}}$ The $K$ magnitude of Ganymede is $u+4$. It is brighter at the $2.22 \mu \mathrm{m}$ narrow filter and considerably fainter at $2.06 \mu \mathrm{m}$. With two $13 \mathrm{~cm}$ diameter apertures and a bandpass of $.08 \mu \mathrm{m}$ the power recieved is $11 \times 10^{-14}$ watts. The present efficiency of the interferometer at $2 \mu \mathrm{m}$ (excluding the detector) is 2.15 and we hope to improve this by a factor of 2 Therefore, the signal at the detector will be $\sim 3 \times 10^{-15}$ watts With $T_{c}=.2$ seconds the noise will be $n \sqrt{1 / T_{c}}=$ $1.6 \times 10^{-16}$ watts The signal is more than an order of magnitude greater than the noise with this bandwidth. It will be possible to measure visibilities of less than 1 with the $2.22 \mu \mathrm{m}$ filter in a few minutes. The $2.06 \mu \mathrm{m}$ filter will require longer integrations

\section{ATMOSPHERIC RESULTS}

As mentioned, the phase of the detector output is a direct measure of the pathlength changes caused by seeing Figure 2 shows the phase versus time at four different wavelengths. The filters used were $K, L, M$, and a $10.2 \mu \mathrm{m}$ filter with $\Delta \lambda=1.4 \mu \mathrm{m}$ The seeing was 2 arcseconds and the baseline was 2 meters, significantly larger than that which will be used for the satellite measurements. The $K, L$, and $M$ data were taken within a few minutes of each other. The $10.2 \mu \mathrm{m}$ data is from earlier in the night. Each point is a determination of the phase from one cycle of the detector signal When the phase changes slowly it is possible to track it for more than $2 \pi$ This is the case for all except the $k$ filter With it there are times when the phase shifts suddenly. When this happens, the absolute phase becomes uncertain by multiples of $2 \pi$.

The $10.2 \mu \mathrm{m}$ phase rarely changes by more than $\pi$ and has a characteristic time scale of a few seconds The phase excursions at $M$ are approximately twice as large $A t L$ and $K$ the excursions are more rapid, having a time constant of less than .2 seconds The variation of seeing with wavelength is not only a change in the amplitude of the phase excursions, but also a change in their frequency. In the future we will obtain more observations to study this effect under a variety of seeing conditions. 


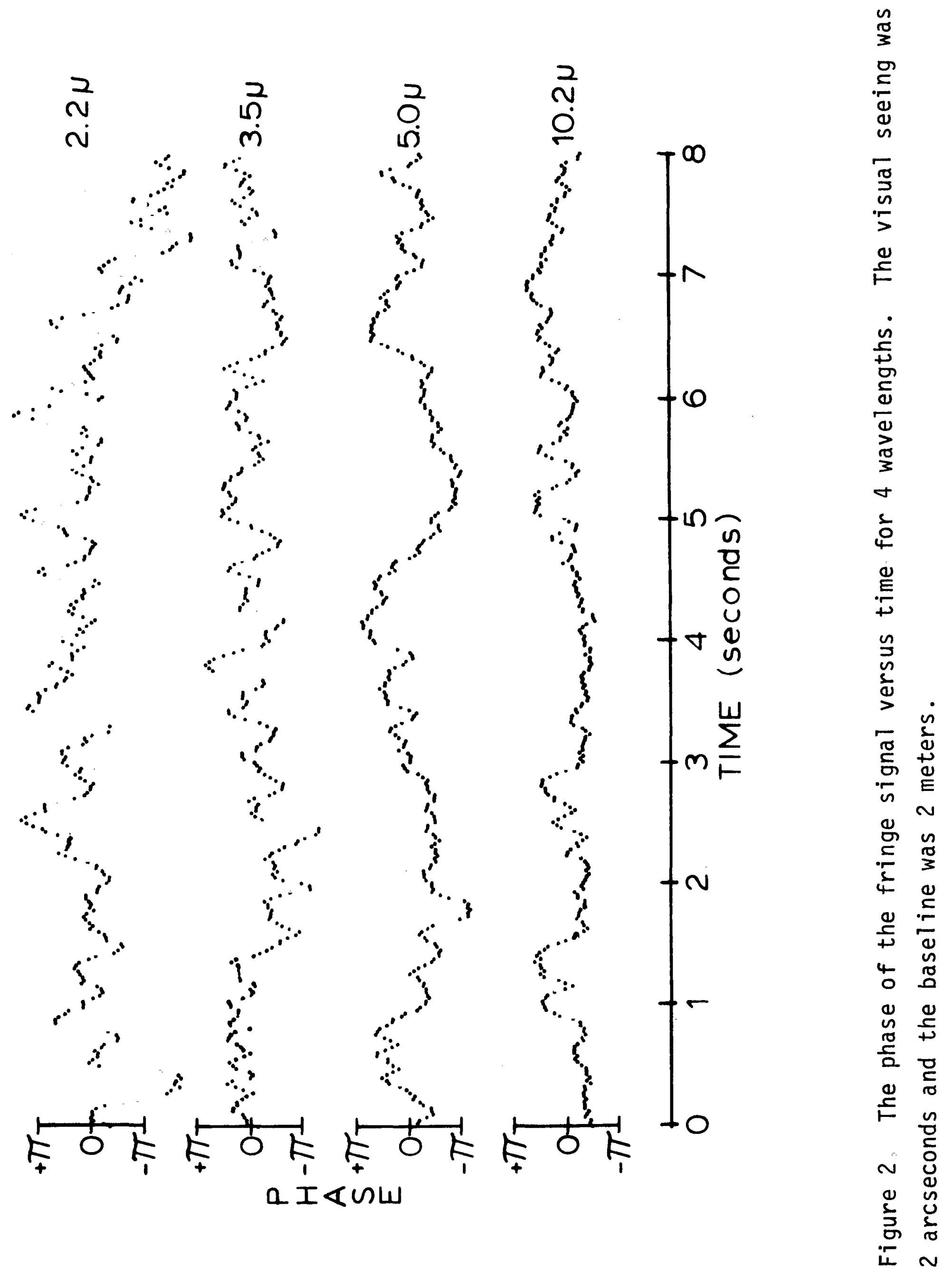




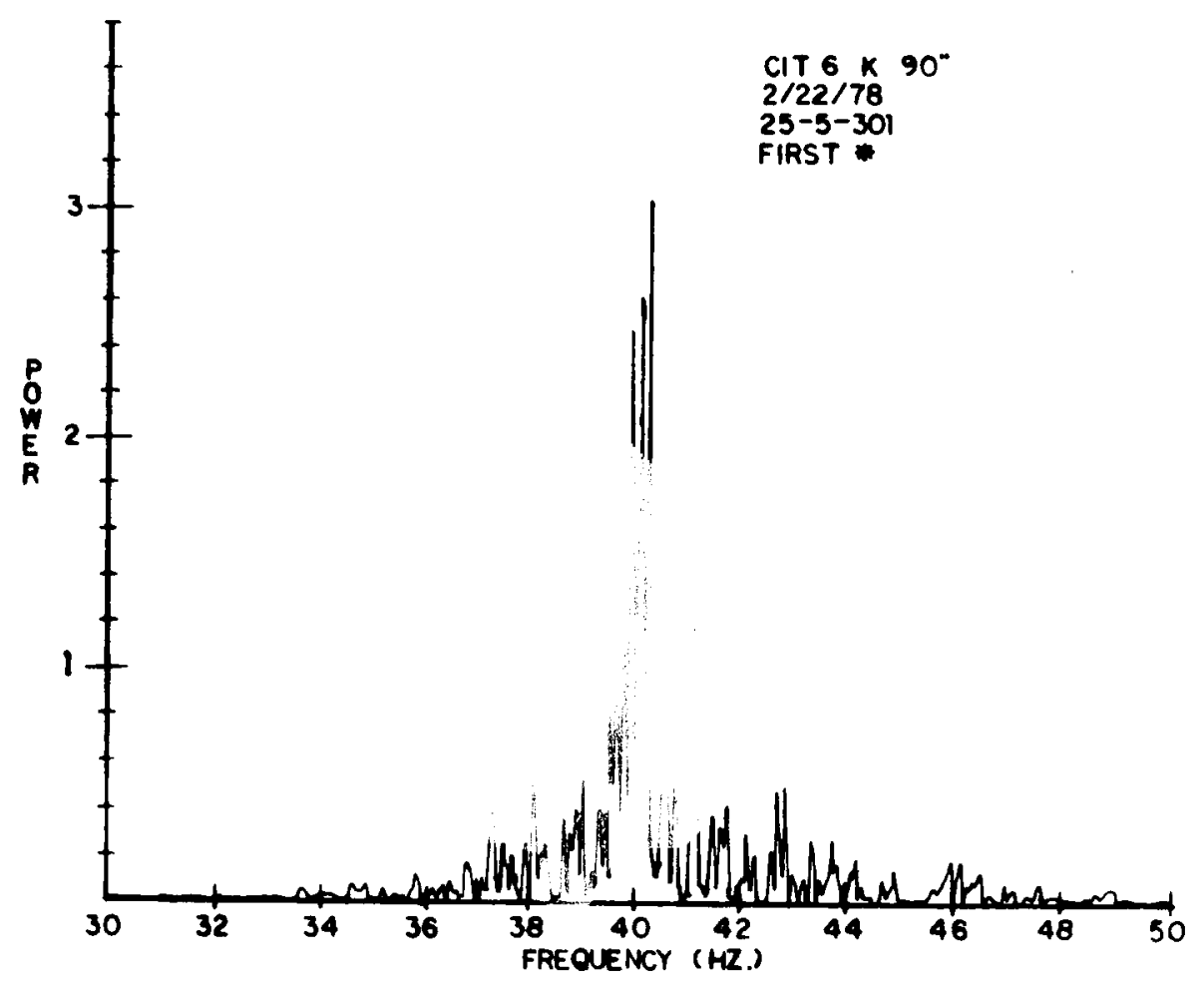

Figure 3. The power spectrum of the fringe signal. The wavelength was $5 \mu \mathrm{m}$ and the baseline was 2 meters.

Figure 3 shows a power spectrum of the fringe signal after being filtered with a 35-50 hz. bandpass. This is at a wavelength of $5 \mu \mathrm{m}$ and is data that was obtained within $1 / 2$ hour of that shown in Figure 2 . As expected from the phase plot in Figure 2 the bandwidth is sightly less than $1 \mathrm{hz}$. When the power spectra of the other wavelengths are computed, the shorter wavelengths are broader. The width does vary with seeing conditions. The narrowest power spectra obtained at $10 \mu \mathrm{m}$ are a few tenths of a hertz wide. This is in contrast to results from the Berkeley heterodyne interferometer where power spectra of less than $.01 \mathrm{hz}$. have been obtained. We cannot explain the discrepancy at this time.

\section{CONCLUSIONS}

Near infrared interferometry can be a useful tool in the study of the Galilean satellites. Such observations will be made within the next few months. In addition, new information concerning infrared seeing can be obtained with this type of interferometer Not only the amplitude but also the frequency of the phase excursions change with wavelength. 
The instrument used for these observations was developed by $\mathrm{Dr}$. McCarthy and Dr Low. Their help has been indispensable I would also like to acknowledge the useful discussions with $\mathrm{Dr}$. G. Rieke and the help of $S$ Allen in reducing the observations The interferometry program is supported by the National Science Foundation

\section{REFERENCES}

1) Michelson, A.A. 1891 , Pub.A.S P , $\underline{3}, 274$.

2) Pilcher, C.B, Ridgway, S.T, and McCord, T.B 1972, Science, 178, 1087.

3) Fink, U, Dekkers, N.H, and Larson, H.P 1973, Ap.J. (Letters), 179, L! 15

4) Lebofsky, L.A. 1977, Nature, 269, 785

5) Fink, U and Larson, H.P 1975, Icarus, 24, 411

6) Rieke, G.H 1977, private communication.

7) Fink, U, Larson, H P., Howell, R, and Rieke, G.H. 1975, B.A.A.S., 7, 386

8) Morrison, D and Cruikshank, D.P 1973, Icarus, 18, 224.

9) Hansen, 0.L. 1973, Icarus, 18, 237

10) Gehrels, T. 1977, in J:A. Burns (ed.), Planetary Satellites, University of Arizona, Tucson, 406.

11) Johnson, M.A. 1974, Ph.D Thesis, University of California, Berkeley

\section{DISCUSSION}

M. Shao: Have you calculated the power spectrum of phase variations and compared it with the Kolmogorov distribution?

R. Howell: No we have not. However, the power is clearly larger at lower frequencies.

C.H. Townes: If you always see rapid fringe variations and never conditions where the fringes vary slowly, then the results you show are different from our experience. At $10 \mu$, your more rapid fluctuations are particularly surprising since our baseline is roughly twice as long. A reason for such 
rapid fluctuations which immediately comes to mind is tracking fluctuations, since any small pointing errors in your system produce fringe shifts, whereas the heterodyne system, using a fixed baseline between two telescopes, is quite insensitive to pointing errors. If one compares $10 \mu$ fringe fluctuations with those at $5 \mu$ or $2 \mu$ there is, in addition to the shorter wavelength, the additional difference of sensitivity to varying water vapour content. The index of refraction of a given density of water vapour is the same as that of the same density of air at $10 \mu$ whereas it is significantly different in other infrared windows. Nevertheless, the fluctuations observed seem rather large to be due to water vapour. Of course, there are a variety of sources of fluctuation due to instrumental effects, some quite esoteric, which an outsider cannot easily evaluate. In general, since it is easy to introduce unnecessary fluctuations and unfortunately very difficult to remove the atmospheric fluctuations, the lower phase fluctuations which we measure must be more nearly those actually due to the atmosphere, and applicable at least to our observing site. If you do see larger fluctuations than we do, they are thus puzzling to me unless they are due to some type of tracking fluctuations, which are of course superimposed on the atmospheric variations. 